\title{
Leitthema
}

Bundesgesundheitsbl 2015 · 58:263-273

DOI 10.1007/s00103-014-2109-y

Online publiziert: 8. Januar 2015

(c) Springer-Verlag Berlin Heidelberg 2014

\author{
M. Pfleiderer ${ }^{1} \cdot 0$. Wichmann $^{2}$ \\ ${ }^{1}$ FachgebietVirusimpfstoffe, Bundesinstitut für Impfstoffe und biomedizinische \\ Arzneimittel, Paul-Ehrlich-Institut, Langen, Deutschland \\ 2 Fachgebiet Impfprävention, Robert Koch-Institut, Berlin, Deutschland
}

\section{Von der Zulassung von Impfstoffen zur Empfehlung durch die Ständige Impfkommission in Deutschland}

\author{
Kriterien zur objektiven Bewertung \\ von Nutzen und Risiken
}

\section{Impfstoffentwicklung und die Rolle der zuständigen nationalen und internationalen Zulassungsbehörden}

Die Entwicklung neuer Impfstoffe ist trotz bedeutender technischer und wissenschaftlicher Fortschritte immer noch eine besondere Herausforderung, die weltweit nur von einigen wenigen hoch spezialisierten und finanziell leistungsfähigen Unternehmen, im weiteren als Impfstoffhersteller bezeichnet, angenommen wird. Dies liegt insbesondere auch daran, dass die Entwicklung neuer Impfstoffe in ein komplexes Netzwerk von Gesetzen und Regularien eingebettet ist. Das bundesdeutsche Arzneimittelgesetz wird von umfangreichen international harmonisierten Regelungen der Arzneimittelgesetzgebung der Europäischen Union (EU) ergänzt, die von Herstellern und Inhabern einer Arzneimittelzulassung beachtet werden müssen $[1,2]$. International operierende Impfstoffhersteller sind darüber hinaus auch noch mit regionalen Besonderheiten konfrontiert, die zusätzliche oder abweichende Regelungen zur Impfstoffzulassung geltend machen können. Dabei sind nicht nur die zum Zeitpunkt der Zulassung gültigen gesetzlichen Regelungen zu beachten, sondern, über das gesamte Produktleben („life cycle") hinweg, auch neu hinzukommende Aufla- gen. Daraus ergibt sich für die Impfstoffhersteller ein komplexes Umfeld, in dem der medizinische Fortschritt mit den Anforderungen der notwendigen regulatorischen Kontrollen zur Sicherstellung der Qualität, Wirksamkeit und Verträglichkeit von Impfstoffen verknüpft werden muss. Den daraus erwachsenden hohen Standards müssen sich auch die zuständigen Behörden stellen. Das Paul-EhrlichInstitut (PEI), Bundesinstitut für Impfstoffe und biomedizinische Arzneimittel, ist wegen seiner Zuständigkeit für Impfstoffe besonders gefordert, durch umsichtiges Handeln seinem gesetzlichen Auftrag - nämlich die Qualität, Sicherheit und Wirksamkeit von Impfstoffen sicherzustellen - gerecht zu werden und dabei gleichzeitig Sorge dafür zu tragen, dass die Entwicklung neuer Impfstoffe nicht durch unangemessene oder schwer nachzuvollziehende regulatorische Vorschriften gefährdet wird.

Da zur Entwicklung und Herstellung neuer Impfstoffe intensive Forschungsarbeit erforderlich ist, kann sich eine für derartige Arzneimittel zuständige Behörde bei ihrer regulatorischen Arbeit nicht darauf beschränken, auf die bestehenden gesetzlichen Regelungen zu verweisen. Eine solche Haltung würde den wissenschaftlichen Fortschritt behindern und in der Folge den Zugang zu neuen Impfstoffen erschweren. Eine zeitgemäße regulato- rische Vorgehensweise erfordert daher eine angemessene Interpretation und Umsetzung der bestehenden Arzneimittelgesetzgebung, mit dem Ziel, ein attraktives Forschungs- und Entwicklungsumfeld zu schaffen und zu bewahren, ohne dabei den oben genannten gesetzlichen Auftrag aus dem Auge zu verlieren. Um derartige Voraussetzungen bieten zu können, muss eine Behörde den gleichen Sachverstand aufweisen wie ein Impfstoffhersteller. Sie muss sich darüber hinaus international so positionieren, dass sie alle wissenschaftlichen und gesetzlichen Vorgaben zur Prüfung und Zulassung von Impfstoffen im Wesentlichen mitbestimmt. Unter diesen Vorgaben ist es möglich, einerseits eine optimale Qualität, Wirksamkeit und Verträglichkeit von Impfstoffen zu gewährleisten und andererseits als öffentlicher Partner der arzneimittelherstellenden Industrie $\mathrm{zu}$ fungieren.

Auf Grundlage dieser Überlegungen ist in den vergangenen Jahren das Konzept der regulatorischen oder auch zulassungs- und prüfungsbegleitenden Forschung entstanden. Dieses fordert, dass sich sowohl eine für Impfstoffe zuständige nationale Behörde wie das Paul-Ehrlich-Institut (PEI) als auch die Europäische Arzneimittelagentur (European Medicines Agency, EMA) als Mediatoren verstehen, die auf der Basis wissenschaftlich fundierter Untersuchungen die Ba- 


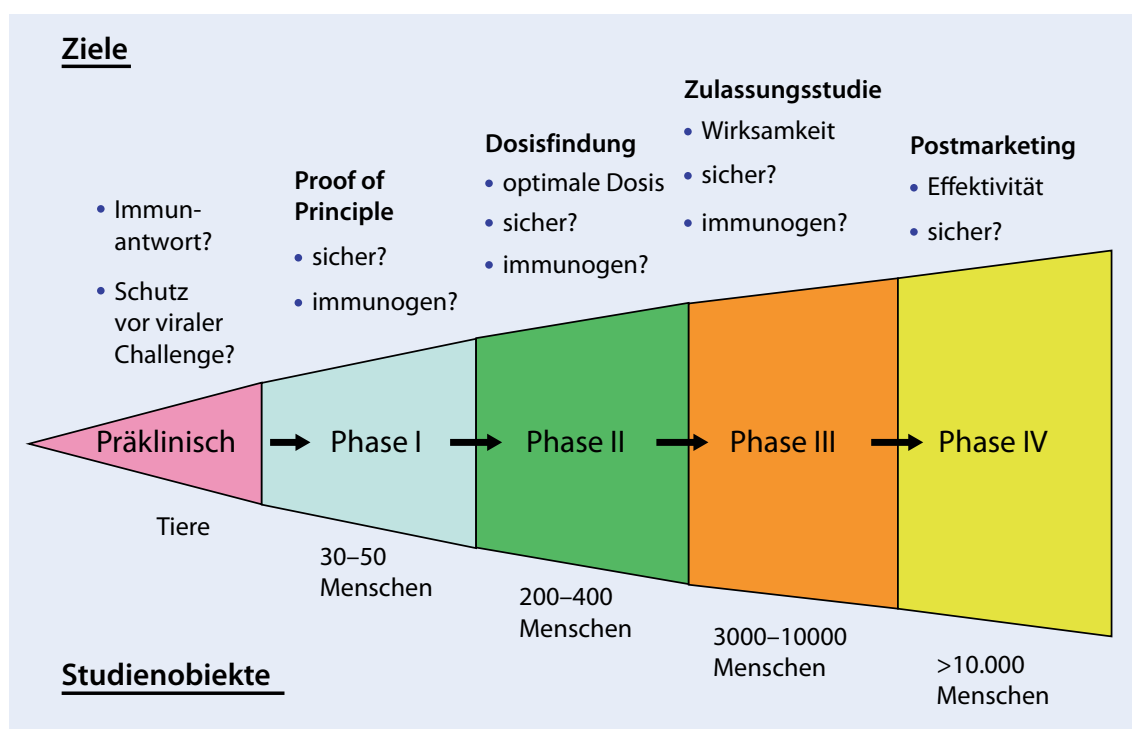

Abb. 1 ॥ Klinische Prüfphasen bei der Entwicklung von Impfstoffen

lance zwischen gesetzgeberischen und regulatorischen Notwendigkeiten und medizinischen Problemstellungen finden. Dabei ist zu bemerken, dass die wissenschaftlichen und regulatorischen Aktivitäten der EMA ebenfalls vom Paul-Ehrlich-Institut, in Zusammenarbeit mit allen anderen EU- Arzneimittelbehörden bzw. Agenturen, begleitet werden. Die regulatorische Forschungskapazität der Arzneimittelbehörden soll Impfstoffherstellern eine ideale Plattform bieten, um die Produktentwicklung und Zulassung effizient vorantreiben zu können. Die regulatorische Forschung entwickelt sich gegenwärtig zu einem anerkannten Wissenschaftszweig, dessen Erkenntnisse in hochwertigen wissenschaftlichen Periodika veröffentlicht werden. Regulatorische Forschung als multidisziplinärer Wissenschaftszweig im Grenzbereich zwischen Medizin, Naturwissenschaften, Pharmazie, Rechtswissenschaften und Verwaltungswesen bezeichnet einen Paradigmenwechsel für das öffentlich-arzneimittelrechtliche Handeln. Ein gut funktionierender Impfstoffmarkt, der den Ansprüchen aller Beteiligten gerecht werden soll, hängt im Wesentlichen von der integrativen Kompetenz der zuständigen Kontrollinstanzen ab.

Wie wichtig zeitgemäßes regulatorisches Management bei der Entwicklung und Prüfung neuer Impfstoffe ist, soll am folgenden Beispiel beschrieben werden.

\section{Erstanwendung von Impfstoffen am Menschen - eine Gratwanderung}

Weit vor der Zulassung stehen die nichtklinische und dann die klinische Prüfung eines Kandidatimpfstoffs. Dass aber der Transfer von Ergebnissen, die in nichtklinischen Prüfungen z. B. am Tiermodell gewonnen wurden, auf den Menschen nicht immer einfach und ungefährlich ist, zeigte der Fall eines zu erprobenden Arzneimittels mit Namen TGN1412 (ein therapeutischer monoklonaler Antikörper): Am 13.03.2006 begann in London eine klinische Studie, in der das Medikament erstmalig am Menschen getestet werden sollte. Mit dem Wirkstoff sollten Erkrankungen behandelt werden, bei denen das Immunsystem - fehlgeleitet - körpereigene Strukturen angreift (sogenannte Autoimmunerkrankungen, wie z. B. die rheumatoide Arthritis - entzündliches Gelenksrheuma oder auch „entzündlicher Rheumatismus"). Acht gesunde erwachsene Probanden (Teilnehmer an einer klinischen Prüfung) nahmen nach entsprechender Aufklärung freiwillig an der Untersuchung teil. Sechs von ihnen erhielten das Prüfpräparat, zwei Studienteilnehmer ein Scheinmedikament (Placebo), um etwaige Nebenwirkungen besser abgrenzen zu können. Innerhalb weniger Stunden entwickelten sich bei den mit TGN1412 behandelten Probanden dramatische Symptome, die einem akuten Schock ent- sprachen [3]. Die vorher durchgeführten, sehr umfangreichen nicht-klinischen Untersuchungen an geeigneten (oder in diesem Fall toxikologisch nicht ausreichend geeigneten) Tiermodellen ließen solche Nebenwirkungen nicht erwarten. Durch den Wirkstoff wurde das Immunsystem der Probanden überstark aktiviert. Eine Aktivierung war zwar der beabsichtigte Effekt, sie fiel jedoch viel stärker aus, als die Tierversuche nahelegten. Die Probanden schwebten tagelang in Lebensgefahr; alle überlebten, jedoch mit teilweise unabsehbaren Langzeitfolgen. Dieses Ereignis führte dazu, dass in den Jahren nach 2006 seitens der Arzneimittelhersteller und Behörden eine Reihe von kritischen Fragen gestellt wurde: Kann man solche innovativen Arzneimittel überhaupt sicher am Menschen testen? Welche Sicherheitsmaßnahmen muss man treffen? Welche Dosis muss man wählen (im Fall der Testung von TGN1412 handelte es sich im Nachhinein um eine Überdosierung)

Aufgrund der Erfahrungen mit TGN1412 und mit ähnlichen Arzneimitteln haben sich im Rahmen der regulatorischen Forschung Prinzipien entwickelt, die helfen sollen, solche Risiken auch bei der Entwicklung neuer Impfstoffe frühzeitig zu erkennen und zu vermeiden. Impfstoffe werden zur Verhinderung von Infektionskrankheiten an Gesunden und nicht zur Behandlung von Krankheiten eingesetzt. Die Risiken, die mit der Anwendung von Impfstoffen verbunden sind, dürfen daher kaum wahrnehmbar sein. Dies gilt im gleichen Maße für die klinischen Prüfphasen (siehe • Abb. 1) und auch für die breite Anwendung nach erfolgter Zulassung.

Die im Folgenden beschriebenen Prinzipien bieten sowohl den Impfstoffherstellern als auch den für Impfstoffe zuständigen Behörden Entscheidungshilfen. Auf ihrer Basis wird die Impfstoffentwicklung nicht behindert, und es werden zudem die Risiken für den Menschen während der klinischen Entwicklungsphase und in der Anwendung nach erfolgter Zulassung weitestgehend reduziert. 
Bundesgesundheitsbl 2015 · 58:263-273 DOI 10.1007/s00103-014-2109-y

(c) Springer-Verlag Berlin Heidelberg 2014

\section{Pfleiderer $\cdot 0$. Wichmann}

\section{Von der Zulassung von Impfstoffen zur Empfehlung durch die Ständige Impfkommission in Deutschland. Kriterien zur objektiven Bewertung von Nutzen und Risiken}

\section{Zusammenfassung}

Impfungen gehören zu den effektivsten Präventionsmaßnahmen der modernen Medizin und haben seit ihrer Einführung im letzten Jahrhundert zu einem drastischen Rückgang, in Einzelfällen sogar zur Eliminierung schwerer Infektionskrankheiten geführt. Die Nutzenbewertung von Impfstoffen weist einige Besonderheiten auf. Dazu gehört, dass Impfstoffe zur Verhinderung von Infektionskrankheiten an Gesunden, Therapeutika hingegen zur Behandlung von bereits Erkrankten eingesetzt werden. Die mit der Anwendung von Impfstoffen verbundenen Risiken dürfen daher - um für den Impfling noch akzeptabel zu sein - nur sehr gering sein. Zum anderen können hohe Impfquoten zu Bevölkerungseffekten (z. B. indirekter Schutz von Ungeimpften) und damit zu einem zusätzlichen Bevölkerungsnutzen führen. Die Zulassung von Impfstoffen geht vornehmlich der Frage nach, ob ein bestimmter Impf- stoff sicher, wirksam und qualitativ hochwertig und sein Nutzen-Risiko-Verhältnis akzeptabel ist. Bei der Entscheidung, ob eine Impfung in ein nationales Impfprogramm aufgenommen bzw. einer bestimmten Risikogruppe empfohlen wird, steht dagegen die Frage im Vordergrund, wie zugelassene Impfstoffe am sinnvollsten in der Bevölkerung angewendet werden (z. B. mit welcher Impfstrategie die meisten Todesfälle, Hospitalisierungen oder Behandlungskosten im Gesundheitssystem reduziert werden können). Bewertungsmethoden für die Nutzen-RisikoAbwägung vor der Erstanwendung von Impfstoffen am Menschen im Rahmen von Zulassungsstudien sind etabliert. Entsprechende Kriterien kommen sowohl bei der Nutzen-Risiko-Abwägung zum Zeitpunkt der Zulassung zur Anwendung als auch während der gesamten Vermarktungsphase. Bei der Entscheidung, ob und wie ein zugelas- sener Impfstoff in ein Impfprogramm integriert wird, kommen Kriterien zum Tragen, die sich von den Zulassungskriterien unterscheiden bzw. über die Kriterien der Sicherheitsund Wirksamkeitsprüfung hinausgehen. Diese Entscheidung sollte zusätzlich auch Bevölkerungseffekte und den lokalen Kontext berücksichtigen (z. B. lokale Epidemiologie, Kosten-Effektivität oder Akzeptanz in der Bevölkerung). Dementsprechend sind nationale Institutionen wie die in Deutschland dafür eingesetzte Ständige Impfkommission (STIKO) etabliert, um global entwickelte Impfstoffe in den nationalen Kontext als Impfstrategien zu integrieren.

\section{Schlüsselwörter}

Impfstoffe · Nutzen-Risiko-Bewertung •

Zulassung $\cdot$ Kosten-Nutzen-Bewertung .

Empfehlung · Impfprogramm .

Paul-Ehrlich-Institut · STIKO

\section{From the licensure of vaccines to the recommendation of the Standing Committee on Vaccination in Germany. Criteria for the assessment of benefits and risks}

\section{Abstract}

Vaccines are among the most effective preventive measures in modern medicine and have led to a dramatic decline and - for a few diseases - even to the elimination of severely infectious diseases. There are some particularities of the risk-benefit assessment of vaccines compared with that of therapeutic drugs. These include the fact that vaccines are applied to healthy individuals with the aim of preventing an infectious disease, while therapeutic drugs are administered to sick people to cure them of an already acquired disease. The acceptable level of risk associated with the application of a vaccine is therefore much lower. In addition, high vaccination coverage can lead to population-level effects (e.g., the indirect protection of unvaccinated individuals) that can confer additional benefits to the population overall. When a marketing authorization application (MAA) for a novel vaccine is evaluated, conclusions are made regarding its quality, safety, and ef- ficacy, and a benefit-risk assessment is carried out accordingly. In contrast, when deciding on the introduction of a new vaccine into a national immunization program or on a recommendation for a specific risk-group, the focus is shifted to considerations of how a licensed vaccine can be best used in a population (e.g., which immunization strategy is most effective in preventing deaths or hospitalizations, or in reducing treatment costs for the health care system). Stringent assessment criteria have been developed that require a robust safety analysis before a new vaccine is administered to humans for the first time in pre-licensure studies. Similarly, criteria are applied for calculating the benefit-risk ratio at the time of the licensure of a new vaccine in addition to during the entire post-licensure period. However, when deciding if and how a licensed vaccine can best be integrated into an existing immunization program, additional criteria are applied that are different, yet complementary to those applied for granting a marketing authorization. These decisions require-in addition to considerations of vaccine quality, vaccine efficacy and safety-conclusions regarding population-level effects combined with an integrative analysis of the local context (e.g., local epidemiology, cost-effectiveness, and acceptance by the population). To serve these objectives, national authorities such as the Standing Committee on Vaccination in Germany (STIKO) have been established to integrate globally developed vaccines into the national context of immunization strategies.

\section{Keywords}

Vaccines - Benefit-risk assessment .

Cost-benefit analyses · Licensure .

Recommendation - Immunization program . Paul-Ehrlich-Institut · STIKO 


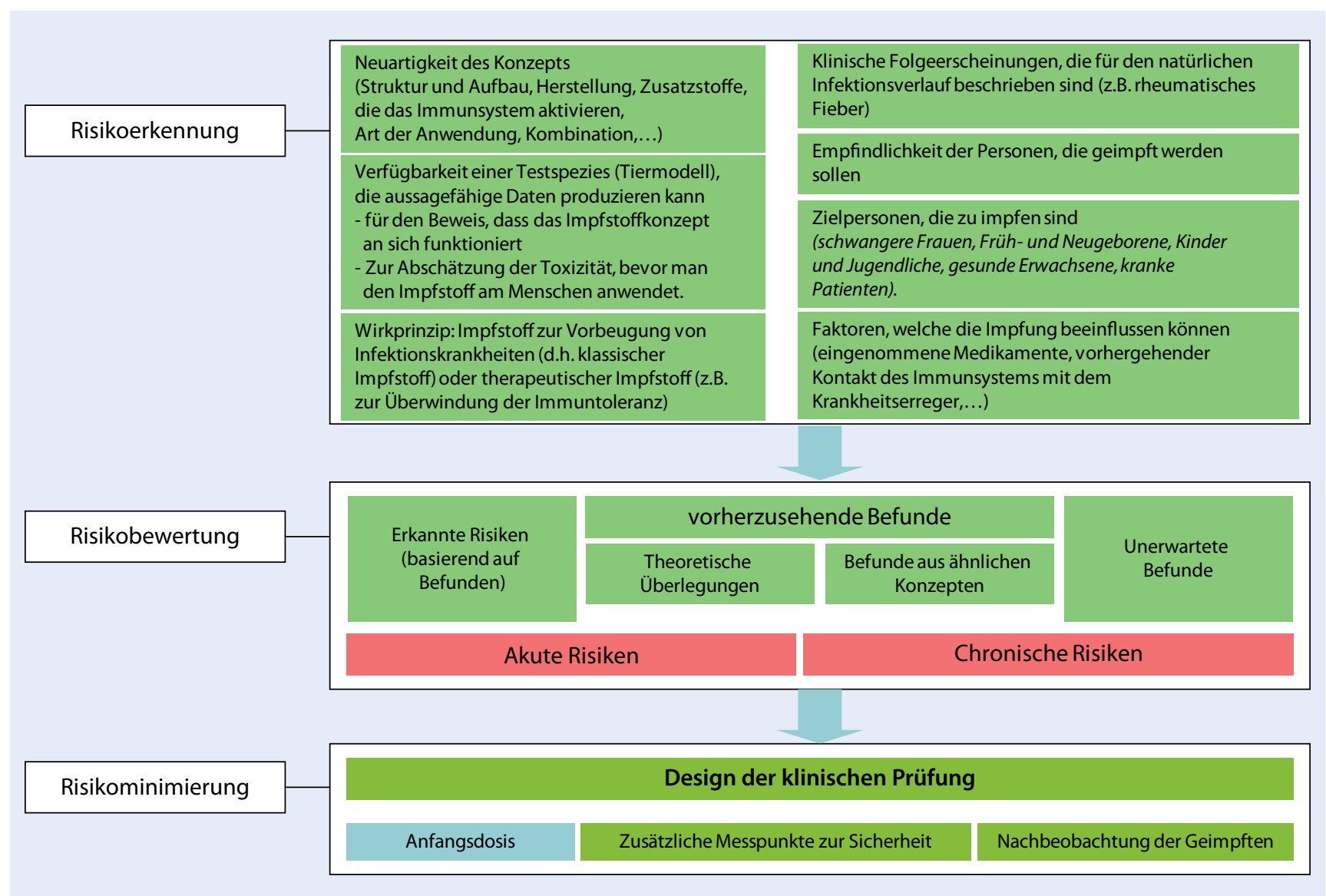

Abb. $2 \Delta$ Risikobewertung für einen neuartigen Impfstoff zur Erstanwendung am Menschen

\section{Erstanwendung von Impfstoffen am Menschen - eine besondere Herausforderung}

Eines der wichtigsten Prinzipien zur Vermeidung von Zwischenfällen wie dem oben beschriebenen ist die Wahl einer geeigneten Startdosis bei der klinischen Erstanwendung eines Arzneimittels/Impfstoffes: Sie muss niedrig genug sein, um eine Gefährdung von Probanden möglichst auszuschließen, aber hoch genug, um noch einen Effekt messen zu können. Bei vielen Arzneimitteln wird man hierfür zunächst nach einem „biologischen Effekt" in nichtklinischen Studien suchen (das kann z. B. die Senkung des Blutdrucks im Tiermodell sein), hieraus dann eine Dosis für den Menschen ableiten und diese dann in der klinischen Prüfung langsam steigern. Dieser Ansatz greift aber bei der Testung von Impfstoffen nicht [4]: Impfstoffe enthalten Bestandteile von Krankheitserregern (z. B. Viren, Bakterien oder Parasiten), die das
Immunsystem gezielt zu ihrer Abwehr aktivieren sollen. Dafür werden die Erreger entweder abgeschwächt oder der Impfstoff enthält nur nichtinfektiöse Erregerkomponenten. Der Geimpften entwickelt dann eine Immunantwort, $d . h$. die verimpften Bestandteile des Krankheitserregers, die Impfantigene, werden vom Immunsystem wie der Krankheitserreger erkannt und neutralisiert. Das schützt den Organismus vor der späteren Infektion und/oder vor einer Erkrankung verursacht durch den „echten“ Krankheitserreger. Anders als bei therapeutischen Arzneimittel steigt die Wirkung eines Impfstoffes, d. h. die Auslösung einer Immunantwort, in der Regel jedoch nicht parallel zur Erhöhung der Dosis: Eine deutliche Immunantwort wird erst ab einer bestimmten Dosis ausgelöst, was die Dosisfindung bei gleichzeitiger Risikoabschätzung in der klinischen Prüfung nach dem Grundsatz der kleinen Startdosis erschwert. Bei herkömmlichen Impfstoffen ist dies kein Problem. Sie gel- ten als ausnehmend sicher. Daher werden bei der Prüfung herkömmlicher Impfstoffe immer Dosen verabreicht, von denen aus vorhergehenden Studien bekannt ist, dass sie eine ausreichende Immunantwort hervorrufen.

Anders sieht das bei neuen Impfstoffkonzepten aus, d. h. wenn z. B. neue Wirkverstärker, sogenannte „Adjuvanzsysteme" geprüft werden sollen, die dazu dienen, eine verbesserte oder spezifischere Immunantwort auszulösen. Mit neuartigen Adjuvanzsystemen oder Immunmodulatoren, deren Entwicklung von den Impfstoffherstellern zügig vorangetrieben wird, kann das menschliche Immunsystem auf vielerlei Art beeinflusst werden, sodass Impfstoffe gegen bislang nicht präventable Infektionskrankheiten wie $\mathrm{z}$. B. AIDS, Hepatitis C oder Malaria in greifbare Nähe rücken. Allerdings birgt der Einsatz immunmodulierender Substanzen immer das Risiko einer systematisch fehlgeleiteten oder überschießenden Immunantwort, die letztendlich zu Verläu- 


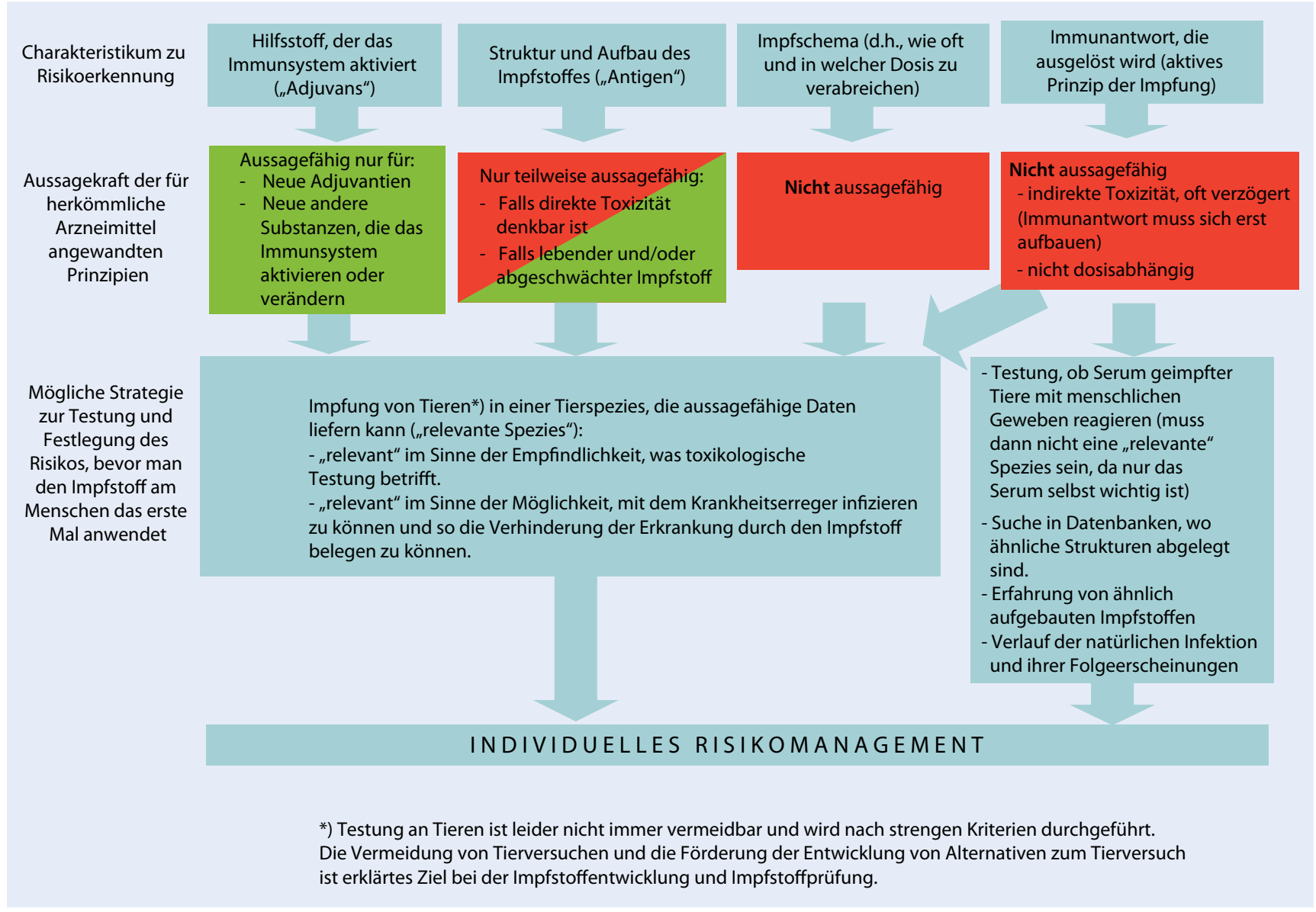

Abb. $3 \Delta$ Faktoren, die man bei der Wahl der Startdosis eines Impfstoffs berücksichtigen sollte, der zur Erstanwendung am Menschen vorgesehen ist

fen führen könnte, wie sie weiter oben für die Prüfsubstanz TGN1412 beschrieben wurden. Würde sich ein solcher Fall im Rahmen der Impfstoffentwicklung wiederholen, wäre das Vertrauen der Öffentlichkeit in Impfstoffe nachhaltig geschädigt.

Aus diesem Grund werden Kriterien und Entscheidungshilfen angewendet, die es erlauben, neue Impfstoffe so zu bewerten, dass bekannte, vermutete sowie unbekannte Risiken erheblich besser kontrolliert, minimiert oder vermieden werden können, als das mit den bisherigen Bewertungsmethoden möglich war (- Abb. 2). Startpunkt ist das Erkennen eines Risikos. Wie $\bullet$ Abb. 2 zeigt, werden hierfür die einzelnen Komponenten des Impfstoffs geprüft. Wirkungen und Nebenwirkungen von Impfstoffen werden von einer Reihe von Faktoren beeinflusst, wie z. B. vom Impfantigen, von den verwendeten Hilfsstoffen, vom Adjuvanzsystem zur zusätzlichen Stimulierung des
Immunsystems, vom Impfschema und letztendlich auch vom Ausmaß und von der Qualität der ausgelösten Immunantwort (• Abb. 3). Dies muss im Einzelfall kritisch hinterfragt werden: Ist die Anwendung des Impfstoffes aufgrund seiner Struktur, seiner Hilfsstoffe oder der Art seiner Anwendung mit einem möglichen Risiko behaftet? Wie verlässlich sind die Daten zur Sicherheit, die man durch Prüfung im Labor oder am Tier erhalten hat?

Die Prüfung an Tieren ist leider nicht immer vermeidbar und wird nach strengen Kriterien durchgeführt. Das Vermeiden von Tierversuchen und die Förderung der Entwicklung von Alternativen sind erklärte Ziele der Hersteller und zuständigen Behörden. Bei der Wahl eines Tiermodells wird man sehr genau darauf achten müssen, ob es erstens aussagefähige Daten zur Toxizität liefern kann und ob es zweitens mit dem Krankheitserreger infiziert werden kann, gegen den sich der Impfstoff wendet. Zur Risikobewertung werden dann sowohl theoretische Überlegungen als auch die konkreten Daten aus den genannten Modellen herangezogen. Danach richtet sich dann, ob zusätzliche Messpunkte zur Sicherheit des Impfstoffs in der ersten klinischen Prüfung am Menschen eingeführt und ob die geimpften Probanden länger als normalerweise nachbeobachten werden müssen.

\section{Regulatorische Forschung als Hilfestellung bei der Impfstoffentwicklung und Impfstoffzulassung}

Die oben dargestellten Überlegungen zur Erstanwendung von Impfstoffen am Menschen haben eine wichtige Lücke ausgefüllt: Im Rahmen von Firmengesprächen, Anträgen zur Genehmigung einer klinischen Prüfung sowie auf der Basis von Erfahrungen bei der Erstanwendung anderer biomedizinischer Arzneimitteln werden die Impfstoffentwickler in einem 


\begin{tabular}{|c|c|}
\hline Zulassung & Empfehlung \\
\hline $\begin{array}{l}\text { Frage: Ist der Impfstoff sicher } \\
\text { und wirksam für den } \\
\text { beabsichtigten Gebrauch? }\end{array}$ & $\begin{array}{l}\text { Frage: Wie kann der zugelassene } \\
\text { Impfstoff am sinnollsten } \\
\text { eingesetzt werden? }\end{array}$ \\
\hline Produktspezifisch: Immer & $\begin{array}{l}\text { Produktspezifisch: Nein, selten } \\
\text { Impfstoffklasse (z.B. präferentiell } \\
\text { Konjugat- oder Lebendimpfstoff) }\end{array}$ \\
\hline Schlüsselkriterien: & Schlüsselkriterien: \\
\hline Produkt-spezifische & $\begin{array}{l}\text { 1) Sicherheit \& Wirksamkeit } \\
\text { verfügbarer Impfstoffe }\end{array}$ \\
\hline 1) Sicherheit & 2) Erwartete indirekte Effekte \\
\hline $\begin{array}{l}\text { 2) Wirksamkeit (inkl. } \\
\text { Immunogenität oder } \\
\text { Effektivität bzgl. } \\
\text { Verhindung klinischer } \\
\text { Endpunkte) }\end{array}$ & $\begin{array}{l}\text { 3) Schwere der Erkrankung und } \\
\text { lokale Epidemiologie (z.B. } \\
\text { Inzidenz/Schwere allgemein } \\
\text { und in bestimmten Alters- } \\
\text { bzw. Risikogruppen) } \\
\text { 4) Integration in Impfkalender }\end{array}$ \\
\hline 3) Qualität & 5) Kosten-Effektivität \\
\hline
\end{tabular}

Abb. $4<$ Hauptfragestellung und Schlüsselkriterien für die Zulassung von Impfstoffen sowie für die anschließende Empfehlung von Impfungen (bzw. Aufnahme einer Impfung in den Nationalen Impfkalender/ in das Nationales Impfprogramm)

multidisziplinären Ansatz beraten und begleitet. Dieser Ansatz ermöglicht die kontinuierliche Nutzen-Risiko-Bewertung von Impfstoffen von der Entwicklung bis zur Zulassung und im Rahmen ihrer Anwendung nach der Zulassung.

Mithilfe dieses wissenschaftlich-regulatorischen Vorgehens werden auch die nationalen Institutionen unterstützt, die sich mit der Erstellung von Impfempfehlungen befassen. Diese können sich weitestgehend auf den mit der Zulassung bestätigten Nutzen von Impfstoffen konzentrieren, da besondere Impfrisiken bereits während des klinischen Entwicklungsprogramms auf ein nicht vermeidbares Minimum reduziert wurden.

\section{Nutzenbewertung des Gebrauchs von zugelassenen Impfstoffen in der Bevölkerung}

In den meisten Industrieländern sind nationale Impfkommissionen etabliert. Es handelt es sich bei ihnen um unabhängige Expertengremien, die auf Basis der besten verfügbaren Evidenz üblicherweise das jeweilige Gesundheitsministerium oder das nationale Public-Health-Institut zur Einführung neuer Impfungen in das nationale Impfprogramm oder zu nationalen Impfstrategien beraten [5]. Die Zulassung von Impfstoffen geht, wie zuvor ausführlich beschrieben, mehr der Frage nach, ob ein spezieller Impfstoff sicher, wirksam und qualitativ hochwertig ist und ob er für die zugelassene Indikation und Bevölkerungsgruppe ein positives Nutzen-Risi-
ko-Verhältnis aufweist. Hingegen steht bei der Entscheidung, ob eine Impfung in ein nationales Impfprogramm aufgenommen bzw. einer bestimmten Bevölkerungsgruppe empfohlen wird, eher die Frage im Vordergrund, mit welcher Impfstrategie zugelassene Impfstoffe am effektivsten in der Bevölkerung angewendet werden. Je nach Epidemiologie und Charakteristika der Infektionskrankheit bzw. des Impfstoffs kann hier beispielsweise auf eine bestimmte Altersgruppe oder Risikogruppe fokussiert werden, was mit einer niedrigeren „number needed to vaccinate“ (d. h. Anzahl an Personen, die geimpft werden müssen, um z. B. einen Todesfall oder eine Hospitalisierung zu verhindern) oder mit einer günstigeren Kosteneffektivität einhergeht als eine nicht zielgerichtete Impfstrategie. Bei Entscheidungen von Impfkommissionen sind also neben den für die Zulassung relevanten Kriterien Sicherheit und Wirksamkeit noch weitere Aspekte zu berücksichtigen ( $\bullet$ Abb. 4). In diesem Kontext wird oft von einer „medizinischepidemiologischen Nutzen-Risiko-Abwägung “ gesprochen (z. B. in der Geschäftsordnung der Ständigen Impfkommission [STIKO] am Robert Koch-Institut [RKI], online verfügbar unter www.stiko.de). Im Folgenden sollen die hier zugrundeliegenden Aspekte beleuchtet werden.

Sowohl bei therapeutischen Arzneimitteln als auch bei Impfstoffen zur Prävention von Infektionskrankheiten erfolgt die Zulassung für eine oder mehrere Indikationen. Je nach Erreger hängt das Risiko für eine Erkrankung bzw. das Risi- ko, bei Infektion einen schweren Krankheitsverlauf $\mathrm{zu}$ entwickeln, unter anderem vom Alter, von möglichen Grunderkrankungen oder auch vom Aufenthaltsort ab. So ist zum Beispiel das Risiko für eine invasive Meningokokkenerkrankung in den ersten Lebensjahren, für einen schweren Influenza-Krankheitsverlauf bei Personen mit chronischen Lungenerkrankungen oder für eine Infektion mit dem Frühsommer-Meningoenzephalitisvirus im süddeutschen Raum deutlich höher als im Vergleich mit anderen Personengruppen oder Regionen [6]. Daher kann eine Impfung mit entsprechend $\mathrm{zu}$ gelassenen Impfstoffen bei einzelnen Personengruppen von unterschiedlich hohem Nutzen sein.

Bei hohen Impfquoten gegen eine Infektionskrankheit reduziert sich die Zirkulation des entsprechenden Erregers in der Bevölkerung, was zu einem indirekten Schutz von ungeimpften Personen führen kann (sog. Herdenschutz). Anders als bei therapeutischen Arzneimitteln kann daher die breite Anwendung einer Impfung in der Bevölkerung neben einem individuellen Nutzen auch einen deutlichen Nutzen für die Gesellschaft haben, z. B. wenn Ausbrüche durch hohe Impfquoten verhindert, vulnerable Personen geschützt (die z. B. aufgrund zu jungen Alters oder einer Grunderkrankung nicht geimpft werden können bzw. bei denen die Impfung nicht gut anspricht) oder eine Infektionskrankheit in einer Region eliminiert werden soll (wie z. B. die Pocken, Masern oder die Kinderlähmung). So wurde in den USA gezeigt, dass die Einführung einer Routine-Impfung von jungen Kindern mit einem 7-valenten Pneumokokken-Konjugatimpfstoff über einen Zeitraum von 5 Jahren nicht nur deutlich die Zahl der Fälle an invasiven Pneumokokken-Erkrankungen in dieser Altersgruppe reduzierte, sondern nochmals doppelt so viele Fälle in höheren (nicht geimpften) Altersgruppen indirekt über den Herdenschutz verhinderte [7]. Aufgrund solcher indirekten Effekte kann für manche Impfungen ein zusätzlicher Bevölkerungsnutzen und folglich ein besonderes öffentliches Interesse an ihnen bestehen. Sie müssen daher bei Entscheidungen über Impfprogramme/Impfempfehlungen und auch bei gesundheitsöko- 
nomischen Evaluationen (siehe unten) berücksichtigt werden.

Während der Bedarf einer erkrankten Person an einem therapeutischen Arzneimittel üblicherweise akut gegeben ist und sie zur Vorstellung beim Arzt motiviert, müssen gesunde Menschen überzeugt werden, sich beim Arzt zur Prävention einer Infektionskrankheit impfen zu lassen. Aufgrund des öffentlichen Interesses an Impfungen und ihres präventiven Charakters werden sie in vielen Ländern in nationale Impfprogramme aufgenommen. Dies geht oft mit einer zentralen Beschaffung des Impfstoffes, einem kostenlosen und niederschwelligen Impfangebot (z. B. in Impfzentren oder Schulimpfungen) und mit Kommunikationsaktivitäten zur Information der Bevölkerung über den Nutzen der Impfung einher. Daher sollten bei der Entscheidung darüber, ob eine Impfung in ein nationales Impfprogramm aufgenommen wird und der Gemeinschaft Kosten verursacht, neben ihrem Nutzen auf Individual- sowie auf Bevölkerungsebene auch die Umsetzbarkeit ihrer Implementierung in das bestehende Programm bzw. ihrer Integration in den existierenden Impfkalender und ihre voraussichtliche Akzeptanz bedacht werden. Bei einem kontinuierlich wachsenden Impfstoffangebot wird es zudem immer mehr $\mathrm{zu}$ einer Herausforderung, in einen ohnehin schon vollen Impfkalender weitere Impfungen zu integrieren, ohne Gefahr zu laufen, dass Eltern oder zu Impfende auf etablierte (und möglicherweise wichtigere) Impfungen zugunsten der neuen Impfung verzichten. Die Abwägung, welche Impfungen für eine Bevölkerung bzw. die öffentliche Gesundheit am wichtigsten sind, gehört zu den wesentlichen Aufgaben nationaler Impfkommissionen.

Im Rahmen der Entscheidungsfindung über eine Impfempfehlung wird neben einer medizinisch-epidemiologischen Risiko-Nutzen-Abwägung in den meisten europäischen Länder auch eine gesundheitsökonomische Evaluation (im engeren Sinne in der Literatur oft auch als Kosten-Effektivitätsanalyse bezeichnet) durchgeführt und dort berücksichtigt [5]. Hierzu werden die Kosten und Effekte eines Szenarios (bspw. vermie- dene Krankheitsfälle, vermiedene Todesfälle, vermiedene Hospitalisierungen oder auch gewonnene Lebensjahre und gewonnene qualitätsadjustierte Lebensjahre [englisch: QALY] [8]), in dem entsprechend den angenommenen Impfquoten ein Teil der Bevölkerung oder einer Kohorte geimpft wird, den Kosten und Effekten eines Szenarios, in dem die entsprechende Impfung nicht verwendet wird, gegenübergestellt. Darauf basierend wird berechnet, welche Kosten im Gesundheitssystem bzw. aus Sicht der Gesellschaft durch das Verhindern bspw. von Erkrankungsfällen aufgrund der Impfung theoretisch entstehen bzw. eingespart werden könnten. Oft fließen die Resultate der gesundheitsökonomischen Evaluation zusammen mit anderen Aspekten in den Entscheidungsprozess von Impfkommissionen über Impfungen bzw. Gesundheitstechnologien mit ein. In europäischen Ländern wie England, der Slowakei, Polen und Irland wird explizit ein sog. QALY-Schwellenwert (Kosten pro gewonnenem QALY, das die Zahlungsbereitschaft einer Gesellschaft bzw. eines Gesundheitssystems widerspiegelt) angewendet, der unterschritten sein muss, damit eine Impfung bzw. eine Gesundheitstechnologie in das nationale Impfprogramm bzw. Gesundheitssystem eingeführt wird. Dies zieht dann in der Regel eine Kostenübernahme durch das jeweilige Gesundheitssystem nach sich. Gesundheitsökonomische Evaluationen können darüber hinaus auch Entscheidungen für eine bestimmte Impfstrategie unterstützen, z. B. darüber, ob die Impfung einer bestimmten Altersgruppe oder Risikogruppe kosteneffektiver (d. h. die Aufwendung von mehr Kosten führt zu höheren Gesundheitseffekten) oder sogar kostensparender ist als die einer anderen Altersgruppe bzw. der gesamten Bevölkerung, oder auch, ob die präferentielle Nutzung einer bestimmten zugelassenen Impfstoffklasse kosteneffektiver ist als die alternative Impfstoffklasse (z. B. Konjugatimpfstoff vs. Polysaccharidimpfstoff). Bei diesen komplexen Modellierungen werden verschiedene Parameter wie bspw. Krankheitsinzidenzen, Impfeffektivität oder erwartete Dauer des Impfschutzes sowie auch der Impfstoffpreis und die (direkten wie auch indirekten) Krankheitskosten berücksichtigt - bzw., wenn nicht verfügbar, angenommen - und in Unsicherheitsanalysen variiert, um zu einer evidenzbasierten (also durch systematisch erhobene Daten und Modellergebnisse gestützten) Entscheidung beizutragen. Üblicherweise sind die in einem nationalen Impfprogramm befindlichen Impfungen kostenfrei. Die übrigen zugelassenen Impfungen, die nicht von einem so hohem öffentlichen Interesse sind, dass sie von der Allgemeinheit finanziert werden, können bei entsprechender individueller Indikation verabreicht werden (müssen dann aber in den meisten Ländern privat bezahlt werden).

Schlussendlich kann es durch die breite Anwendung einer Impfung in seltenen Fällen auch zu negativen Effekten auf Bevölkerungsebene kommen, wie z. B. zu einer Verschiebung der Krankheitslast hinein in höhere Altersgruppen (insbesondere nach Einführung von Kinderimpfprogrammen ohne Schließung der Immunitätslücken bei Älteren - siehe z. B. Masern in Deutschland [9]), zu einem Replacement von im Impfstoff befindlichen Erregerserotypen mit nicht im Impfstoff befindlichen Typen (z. B. bei der Pneumokokkenimpfung [10]) oder zum hypothetischen Anstieg der Herpes-zoster-Inzidenz unter ungeimpften Älteren nach Einführung einer routinemäßigen Impfung von Kindern gegen Varizellen aufgrund fehlender Booster-Infektionen bei niedrigerer Varizella-zoster-Viruszirkulation [11]. Solch negative Effekte können den Nutzen einer Impfung auf Bevölkerungsebene reduzieren und müssen ebenfalls in die abschließende Bewertung einfließen.

\section{Die Rolle und Arbeitsweise der Ständigen Impfkommission in Deutschland}

In Deutschland gibt die Ständige Impfkommission (STIKO) beim Robert Koch-Institut (RKI) gemäß $₫ 20$ Abs. 2 des Infektionsschutzgesetzes (IfSG) Empfehlungen zur Durchführung von Schutzimpfungen. Zu den Hauptaufgaben der STIKO gehört die Aufstellung eines Impfkalenders für Säuglinge, Kinder und Erwachsene. Diese Aufga- 
Tab. 1 Fragenkatalog der Ständigen Impfkommission (STIKO). Fragekomplexe, die standardisiert bei der Entwicklung einer neuen Impfempfehlung durch die STIKO bearbeitet werden müssen [14]

\begin{tabular}{|c|c|}
\hline Fragenkomplex & Erläuterung \\
\hline Fragenkomplex zum Erreger & $\begin{array}{l}\text { Z. B. Charakteristika des Erregers, Vorkommen von Erreger- } \\
\text { Subtypen }\end{array}$ \\
\hline Fragenkomplex zur Zielkrankheit & $\begin{array}{l}\text { Z. B. Inzidenz, Hospitalisierungsrate, Mortalität, Seroprävalenz, } \\
\text { Komplikationen und Behinderung nach Erkrankung, ggf. nach } \\
\text { Risikogruppen/Serotypen sowie Wahrnehmung in der Be- } \\
\text { völkerung }\end{array}$ \\
\hline $\begin{array}{l}\text { Fragenkomplex zum Impfstoff/zu } \\
\text { Impfstoffen }\end{array}$ & $\begin{array}{l}\text { Z. B. Wirksamkeit der Verhinderung definierter Endpunkte, } \\
\text { Sicherheit, Dauer des Schutzes, Notwendigkeit von Booster- } \\
\text { Impfungen, Koadministration mit anderen Impfstoffen, Quali- } \\
\text { tät des Korrelats für Schutz, falls nur Immunogenitätsstudien } \\
\text { zur Verfügung stehen }\end{array}$ \\
\hline Fragenkomplex zur Impfstrategie: & $\begin{array}{l}\text { Z. B.: Welches Impfziel soll erreicht werden? Welche Faktoren } \\
\text { sprechen gegen ein Erreichen der Impfziele? Mögliche positive } \\
\text { bzw. negative Auswirkungen einer Impfempfehlung auf Be- } \\
\text { völkerungsebene? Welche Impfquoten sind notwendig, um } \\
\text { positive Bevölkerungseffekte zu erreichen? }\end{array}$ \\
\hline $\begin{array}{l}\text { Fragenkomplex zur Implementie- } \\
\text { rung einer Impfempfehlung }\end{array}$ & $\begin{array}{l}\text { Z. B.: Ist eine mögliche Impfempfehlung umsetzbar? Akzep- } \\
\text { tanz der Impfung in der Bevölkerung oder der Ärzteschaft? } \\
\text { Kosten-Effektivitätsanalyse. Alternative Maßnahmen für das } \\
\text { Erreichen des Impfziels im Vergleich zur Impfung? Existieren } \\
\text { Monitoring-Systeme zur Evaluation der Impfung bzw. der } \\
\text { Impfempfehlung? }\end{array}$ \\
\hline
\end{tabular}

be wurde bereits in der Begründung zum Gesetzentwurf der Bundesregierung zum Seuchenneuordnungsgesetz im Jahr 2000 formuliert [12, 13]. Zudem soll die Kommission festlegen, „welche Schutzimpfungen die gesamte Bevölkerung oder bestimmte Bevölkerungsgruppen (Risikogruppen) zu welchem Zeitpunkt und in welchen Intervallen erhalten sollten. Entsprechend der Zielsetzung des IfSG sind dabei insbesondere solche Schutzimpfungen relevant, die von allgemeiner gesundheitspolitischer Bedeutung für die Gesundheit der Bevölkerung sind. Dies ist z. B. der Fall, wenn es sich um Schutzimpfungen mit breiter Anwendung und erheblichem Wert für die Gesundheit der Bevölkerung handelt und bundesweit hohe Immunisierungsraten angestrebt werden, wenn so gut verträgliche und finanzierbare Schutzimpfungen gegen bestimmte Krankheiten zur Verfügung stehen, dass diese Krankheiten aus sozialen und volkswirtschaftlichen Gründen so weit wie möglich zurückgedrängt werden sollten [...] oder auch wenn es um den gezielten Schutz besonders gefährdeter Bevölkerungsteile geht [...]“ [12].

Die STIKO wurde im Jahr 1972 eingerichtet. Ihre Tätigkeit wird von der Geschäftsstelle im Robert Koch-Insti- tut koordiniert und z. B. durch systematische Analysen der Fachliteratur unterstützt. Entsprechend ihrer Geschäftsordnung legt die STIKO ihr methodisches Vorgehen bei den Prüfungen und Bewertungen nach dem Stand der Wissenschaft selbst fest. 2011 verabschiedete sie eine neue Methodik (sog. Standardvorgehensweise), die in wesentlichen Punkten der Methodik der evidenzbasierten Medizin folgt [14]. So wird bei der Entwicklung einer neuen Impfempfehlung ein Fragenkatalog mit spezifischen Fragestellungen systematisch bearbeitet (• Tab. 1). Die Evidenz zur Wirksamkeit und Sicherheit der Impfung wird üblicherweise in einem systematischen Review zusammengetragen und die Qualität der Evidenz mittels der Methode der Arbeitsgruppe „Grading of Recommendations Assessment, Development and Evaluation“ (kurz: GRADE) bewertet [15]. Hierbei können sowohl Daten aus Zulassungsstudien (üblicherweise randomisierte kontrollierte Phase-1- bis Phase-3-Studien) als auch aus der Post-Marketing-Phase (üblicherweise Beobachtungsstudien) in die Evidenzbewertung eingehen [14]. Abbildung 5 gibt den Ablauf der systematischen Reviews und den Eingang weiterer Kriterien aus dem STIKO-Fragenkatalog in die abschließen- de Risiko-Nutzen-Bewertung der STIKO wider.

Die STIKO-Empfehlungen entfalten keine unmittelbare rechtliche Wirkung. Die obersten Landesgesundheitsbehörden entscheiden jedoch gemäß $\$ 20$ Abs. 3 IfSG auf Grundlage der STIKO-Empfehlungen über ihre öffentlichen Empfehlungen, die u. a. für das soziale Entschädigungsrecht in $\$ 60$ IfSG relevant sind. Ferner sind die STIKO-Empfehlungen seit 2007 gemäß $\$ 20$ d Abs. 1 des Fünften Buches Sozialgesetzbuch (SGB V) Grundlage für die Schutzimpfungsrichtlinien des Gemeinsamen Bundesausschusses (G-BA), in denen die Einzelheiten zu Voraussetzungen, Art und Umfang von Schutzimpfungen als Pflichtleistung der gesetzlichen Krankenversicherung (GKV) bestimmt werden „unter besonderer Berücksichtigung der Bedeutung der Schutzimpfungen für die öffentliche Gesundheit“ (• Abb. 6). Aufgrund dieser im Rahmen des GKV-Wettbewerbsstärkungsgesetzes vorgenommenen $\mathrm{Ge}$ setzesänderung kommt der STIKO eine wesentliche neue Rolle zu, die ihr mehr als noch zuvor eine hohe wissenschaftliche Qualität ihrer Entscheidungen abverlangt und eine Unabhängigkeit von kommerziellen Interessen. Da die STIKO bei der Risiko-Nutzen-Bewertung die Bevölkerungsperspektive mit einbezieht, findet nicht jede mit einem zugelassenen Impfstoff mögliche Impfung Eingang in die STIKO-Empfehlungen. Unabhängig davon liegt es im Ermessen eines jeden Arztes, einem Patienten entsprechend seiner individuellen (gesundheitlichen) Situation auch eine von der STIKO nicht empfohlene Impfung vorzuschlagen, wenn dies im Einzelfall eine sinnvolle Maßnahme ist [16]. Während sich die STIKO explizit gegen die Aufnahme einer Impfung in die Impfempfehlungen entscheiden kann, kann andererseits auch die Situation entstehen, dass für eine Empfehlung zur breiten Anwendung eines neu zugelassenen Impfstoffs in der Bevölkerung wesentliche Evidenz (noch) nicht vorliegt. In diesem Fall kann die STIKO eine Stellungnahme veröffentlichen, in der darauf hingewiesen wird, dass eine erste Beschäftigung mit dem Thema stattfand, aber in Ermangelung von Daten, die für die Entscheidungsfindung wesentlich 


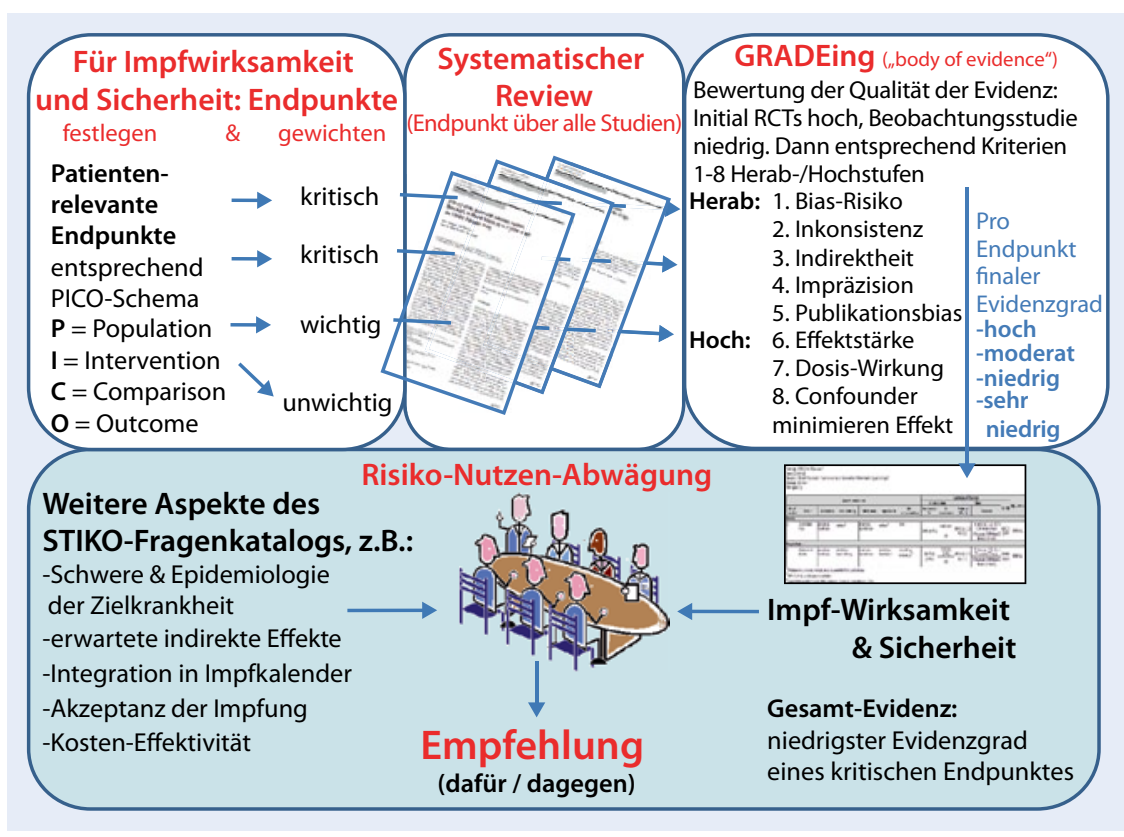

Abb. $5 \Delta$ Vorgehen der Ständigen Impfkommission (STIKO) bei der Bewertung der vorliegenden Evidenz und Berücksichtigung verschiedener Schlüsselfaktoren. RCT Randomisierte kontrollierte Studie, Publ.-Bias Publikations-Bias. (Abbildung adaptiert nach GRADE) [19]

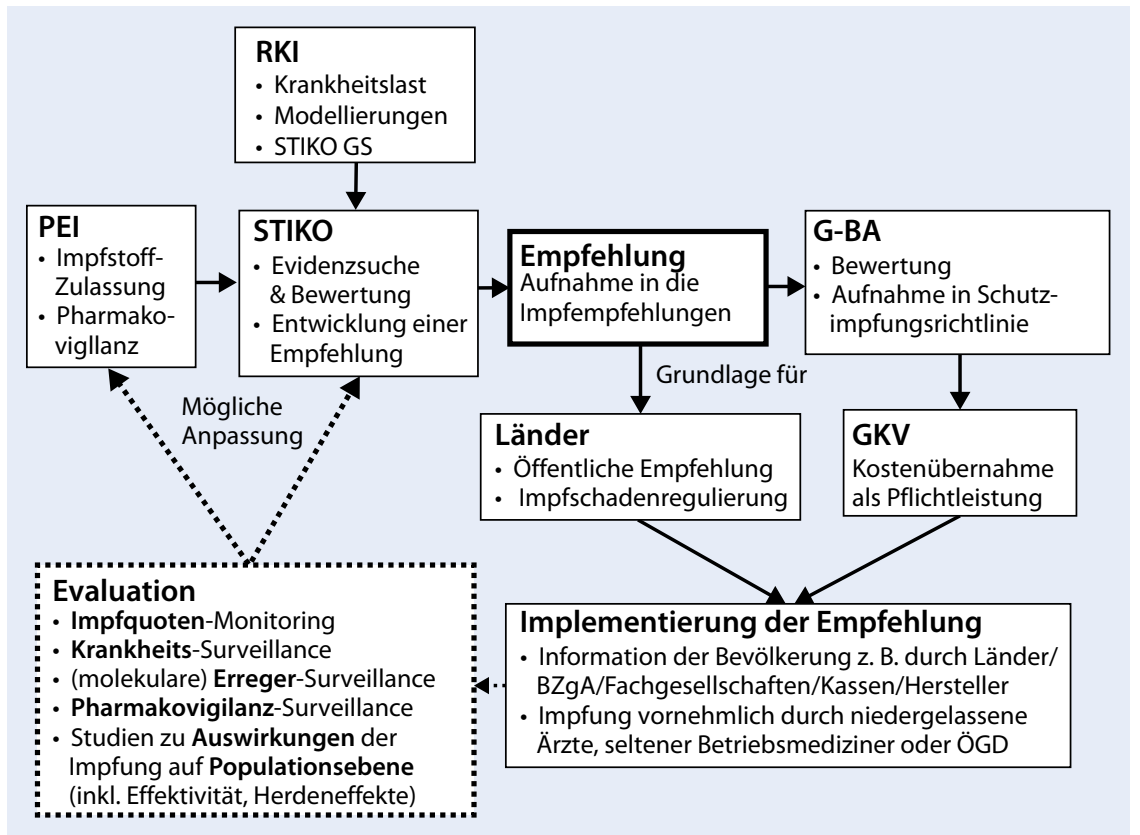

Abb. $6 \Delta$ Das Impfsystem und seine Akteure in Deutschland. PEI Paul-Ehrlich-Institut, RKI Robert Koch-Institut, GS Geschäftsstelle, STIKO Ständige Impfkommission, G-BA Gemeinsamer Bundesausschuss, GKV Gesetzliche Krankenversicherungen, BZgA Bundeszentrale für Gesundheitliche Aufklärung, ÖGD Öffentlicher Gesundheitsdienst. [Adaptiert nach 18]

sind, keine abschließende Entscheidung getroffen werden konnte. Dies geschah z. B. aktuell bei einem neuen Impfstoff gegen Meningokokken B [17].

\section{Notwendigkeit der Evaluierung von Impfungen nach der breiten Anwendung (Post-Marketing-Monitoring)}

Zum Zeitpunkt einer Entscheidung darüber, ob eine Impfung eingeführt bzw. emp- fohlen wird, liegen zu manchen Parametern (z. B. Dauer des Impfschutzes, indirekte Effekte) keine ausreichenden Daten vor. Um z. B. den möglichen Nutzen der Impfung abhängig von den erreichten Impfquoten vorhersagen zu können (z. B. als Anzahl verhinderter Fälle, verhinderter Hospitalisierungen oder verhinderter Todesfälle), kann man sich aber mathematischer Modelle bedienen. Mit ihrer Hilfe können zu erwartende Effekte unter Berücksichtigung verschiedener Szenarien und Unsicherheiten projiziert werden. Diese Modellierungen bieten eine wesentliche Hilfestellung zum Verständnis komplexer Interaktionen zwischen verschiedenen Parametern, die sich auf den Nutzen einer Impfung in der Bevölkerung auswirken können.

Nach der breiten Anwendung einer Impfung in der Bevölkerung müssen jedoch Systeme vorhanden sein, die eine kontinuierliche Evaluation der jeweiligen Impfung bzw. der durch sie verursachten Effekte auf die Zielkrankheit erlauben (• Abb. 6). Dazu gehören ein Monitoring der Krankheitslast in den verschiedenen Altersgruppen vor und nach Einführung der Impfung (z. B. auf Basis von IfSG- Meldedaten), eine kontinuierliche Erhebung der Impfquoten (zur Beurteilung der epidemiologischen Effekte), eine Pharmakovigilanz-Surveillance (die auch sehr seltene unerwünschte Arzneimittelwirkungen detektiert) und eine molekulare Surveillance des Erregers (die z. B. mögliche Escapemutanten oder Replacementphänomene aufdeckt). Außerdem müssen gezielte Beobachtungsstudien zur Impfeffektivität die Frage überprüfen, ob die in den Zulassungsstudien unter idealen Bedingungen bzw. in selektiven Studienpopulationen beobachtete Wirksamkeit dann auch in einem Impfprogramm eine entsprechende Effektivität in der Bevölkerung zeigt (• Abb. 6). Post-Marketing-Studien zur Impfeffektivität sind insbesondere dann von Bedeutung, wenn die Zulassung eines Impfstoffs nur auf Immunogenitätsstudien beruhte (was z. B. bei seltenen Erkrankungen wie Meningokokken alternativlos ist). Das Monitoring ermöglicht, Empfehlungen oder sogar die Zulassung entsprechend zu adaptieren, um den Nutzen einer Impfung in der Bevölkerung zu optimieren (z. B. die Verabreichung einer zweiten Dosis bei der Va- 
rizellenimpfung oder die Reduzierung der Anzahl an Dosen in einem Impfschema bei der HPV-Impfung oder der Pneumokokken-Konjugatimpfung bei Kindern).

\section{Fazit}

Auch wenn die Prinzipien, die bei der Impfstoffzulassung bzw. im Rahmen der Empfehlung von Impfungen angewendet werden durchaus unterschiedlich sind, so haben sie dennoch ein gemeinsames Ziel: es möglich zu machen, dass den Geimpften bzw. der Bevölkerung, in der die Impfung im Rahmen eine Programms durchgeführt wird, der größtmögliche Nutzen einer Impfung bei gleichzeitiger Minimierung von Impfrisiken zukommt. Angesichts der weltweit vergleichsweise geringen Zahl an Impfstoffherstellern sind die Entwicklung neuer Impfstoffe und die Art und Weise, wie diese geprüft und zugelassen werden, immer von überregionalen, wenn nicht sogar globalen Überlegungen bestimmt. Dies ist erforderlich, um einerseits den weltweit einheitlichen Zugang zu modernen Impfstoffen zu gewährleisten, und andererseits um zu vermeiden, dass über ein und denselben Impfstoff unterschiedliche wissenschaftliche Schlussfolgerungen gezogen werden. Entsprechend arbeiten die großen europäischen Zulassungsbehörden (EMA und nationale Behörden) schon seit Langem intensiv zusammen, vermehrt auch unter Beteiligung der WHO und im Rahmen von wissenschaftlichen und regulatorischen Beratungen, immer unter Beteiligung der Impfstoffhersteller. Nur so wird gewährleistet, dass neue Impfstoffe den weltweit gültigen Standards genügen und nach ihrer Zulassung erfolgreich in bestehende Impfkalender integriert werden können. Die Umsetzung dieses letzten Schrittes vor der Anwendung neuer Impfstoffe ist meist Aufgabe nationaler Institutionen, in Deutschland der STIKO, die sich zur Ausübung ihrer Tätigkeit größtenteils auf nationale und weniger auf internationale Vorgaben beziehen müssen. Dies liegt nicht zuletzt daran, dass Aufbau und Funktionalität nationaler Gesundheitssysteme viel weniger standardisierbar sind als wissenschaftliche und regulatorische Vorgaben zur Impfstoffentwicklung, -prüfung und -zulassung, da hier lokale kontextsensible Aspekte zu berücksichtigen sind (wie z. B. die Epidemiologie einer Infektionskrankheit, Kosten im Gesundheitssystem oder Werte/Präferenzen in der Bevölkerung). Diese Parameter, einschließlich der gesundheitsökonomischen Evaluationen, werden von den Zulassungsbehörden bei ihren Entscheidungen nicht oder nur am Rande berücksichtigt. Entscheidungen über nationale Impfempfehlungen können aber nur auf Grundlage des lokalen Kontextes gefällt werden und folglich auch nur von regionalen bzw. nationalen Institutionen, die über die erforderliche Kompetenzen verfügen, um globale Impfstoffkonzepte in nationale Impfstrategien zu integrieren. Daher ist auch eine frühzeitige Konsultation von nationalen Public-Health-Instituten bzw. Impfkommissionen sinnvoll, um bereits vor Zulassung eines neuen Impfstoffs den Bedarf an Daten für Entscheidungen zu nationalen Impfprogrammen zu identifizieren und diese zu generieren. Auf diese Weise wird verhindert, dass nach der Zulassung eines neuen Impfstoffs Entscheidungen über seine mögliche Aufnahme in ein nationales Impfprogramm zusätzlich verzögert werden oder Entscheidungsträger gezwungen werden, diese auf Basis qualitativ niedriger Evidenz zu treffen.

\section{Korrespondenzadresse}

\section{Pfleiderer}

FachgebietVirusimpfstoffe, Bundesinstitut für Impfstoffe und biomedizinische Arzneimittel Paul-Ehrlich-Institut, Paul-Ehrlich-Straße 51-59 63225 Langen

michael.pfleiderer@pei.de

Danksagung. Die Autoren möchten sich bei Frau Dr. Anette Siedler und Herrn Dr. Berhard Ultsch (beide Robert Koch-Institut) für die hilfreichen Kommentare insbesondere zu den Abschnitten zur Entwicklung von Impfempfehlungen bzw. zur STIKO sowie zu gesundheitsökonomischen Aspekten bedanken.

\section{Einhaltung ethischer Richtlinien}

Interessenkonflikt. Dr. rer. nat. Michael Pfleiderer ist Leiter des Fachgebiets Virusimpfstoffe am Paul-Ehrlich-Institut. Bei der europäischen Arzneimittelagentur (EMA) ist er der Vorsitzende der Vaccine Working Party (VWP) des Ausschusses für Humanarzneimittel (CHMP) sowie Mitglied der CHMP Biologics Working Party (BWP). Beide Gruppierungen sind maßgeblich in die Entscheidungsprozesse zur Zulassung neuer Arzneimittel eingebunden.

PD Dr. med. Ole Wichmann ist Leiter des Fachgebiets Impfprävention am Robert Koch-Institut. In dem Fachgebiet ist auch die Geschäftsstelle der Ständigen Impfkommission angesiedelt.

\section{Literatur}

1. Arzneimittelgesetz http://www.gesetze-im-internet.de/amg_1976/.Zugegriffen: 17.Dez. 2014

2. EU Legislation - Eudralex http://ec.europa.eu/ health/documents/eudralex/index_en.htm

3. Stebbings R, Poole S, Thorpe R (2009) Safety of biologics, lessons learnt from TGN1412. Curr Opin Biotechnol 20(6):673-677

4. Goetz KM, Pfleiderer M, Schneider CK (2010) Firstin-human clinical trials with vaccines - what do regulators want? Nat Biotechnol 28(9):910-916

5. Nohynek H, Wichmann O, D' Ancona F (2013) VENICE National Gatekeepers. National Advisory Groups and their role in immunization policy-making processes in European countries. Clin Microbiol Infect 19(12):1096-1105

6. Robert Koch-Institut. RKI-Ratgeber für Ärzte. www. rki.de/ratgeber

7. Centers for Disease Control and Prevention (CDC) (2005) Direct and indirect effects of routine vaccination of children with 7-valent pneumococcal conjugate vaccine on incidence of invasive pneumococcal disease - United States, 1998-2003. MMWR Morb Mortal Wkly Rep 54(36):893-897

8. Postma MJ, Westra TA, Quilici S, Largeron N (2013) Economic evaluation of vaccines: specificities and future challenges illustrated by recent European examples. Expert Review of Vaccines 12:555-565

9. Siedler A, Mankertz A, Feil F, Ahlemeyer G, Hornig A, Kirchner M, Beyrer K, Dreesman J, Scharkus S, Marcic A, Reiter S, Matysiak-Klose D, Santibanez S, Krause G, Wichmann $O$ (2011) Closer to the goal: efforts in measles elimination in Germany 2010. J Infect Dis 204(Suppl 1):S373-S380

10. Miller E, Andrews NJ, Waight PA, Slack MP, George RC (2011) Herd immunity and serotype replacement 4 years after seven-valent pneumococcal conjugate vaccination in England and Wales: an observational cohort study. Lancet Infect Dis 11(10):760-768

11. Edmunds WJ, Brisson M (2002) The effect of vaccination on the epidemiology of varicella zoster virus. J Infect 44(4):211-219

12. Bales S, Baumann HG, Schnitzler N (2003) Infektionsschutzgesetz, Kommentar und Vorschriftensammlung. In: Bales S, Baumannn HG, Schnitzler N (Hrsg), 2. Aufl. W. Kohlhammer, Stuttgart

13. Gesetzentwurf der Bundesregierung. Entwurf eines Gesetzes zur Neuordnung seuchenrechtlicher Vorschriften, BT-Drs. 14/2530, S 71 
14. Standardvorgehensweise (SOP) der Ständigen Impfkommission (STIKO) für die systematische Entwicklung von Impfempfehlungen. Version 2.0 vom 06.02.2014. www.stiko.de >Aufgaben und Methodik. Zugegriffen: 24. März 2014

15. Guyatt GH, Oxman AD, Schünemann HJ, Tugwell P, Knottnerus A (2011) GRADE guidelines: a new series of articles in the Journal of Clinical Epidemiology. J Clin Epidemiol 64:380-382

16. Ständige Impfkommission Empfehlungen der Ständigen Impfkommission (STIKO) am Robert Koch-Institut/Stand: August 2014. Epidemiol Bull 34/2014

17. Ständige Impfkommission (STIKO) am RKI (2014) Aktualisierte Stellungnahme zum Stand der Bewertung des neuen Meningokokken-B-Impfstoffs. Epidemiol Bull 36/2014

18. Wichmann O (2012) Leitlinien für Schutzimpfungen. In: Spiess H, Heininger U, Jilg W (Hrsg) Impfkompendium, 7. Aufl. Georg Thieme Verlag, Stuttgart

19. Guyatt G, Oxman AD, Akl EA, Kunz R, Vist G, Brozek J, Norris S, Falck-Ytter Y, Glasziou P, DeBeer H, Jaeschke R, Rind D, Meerpohl J, Dahm P, Schünemann HJ (2011) GRADE guidelines: 1. Introduction-GRADE evidence profiles and summary of findings tables. J Clin Epidemiol 64(4):383-394 